TRABAJO EN PROGRESO

\title{
Diseño de una aplicación móvil con realidad aumentada para coadyuvar en el proceso de aprendizaje matemático
}

\author{
Eduardo Sosa-Jiménez, José López-Martínez, \\ Victor Chi-Pech, Omar Sosa-Tzec
}

Publicado: 31 Octubre 2018

\begin{abstract}
Resumen
En México, el desempeño de los estudiantes en las asignaturas relacionadas con las matemáticas es muy bajo, aún siendo las matemáticas parte esencial en nuestra sociedad. En los últimos años, las instituciones educativas han utilizado las tecnologías de la información y comunicación con el objetivo de disminuir dicha problemática, al generar herramientas que sean de apoyo en el aprendizaje de los estudiantes. La Realidad Aumentada (RA), es una tecnología que permite sobreponer información a la observada en el mundo real con el objetivo de que el usuario obtenga mayor conocimiento de su entorno. En este trabajo se presenta un diseño de una aplicación móvil como una herramienta para facilitar el aprendizaje matemático a través del uso de RA en estudiantes de nivel medio superior en el estado de Yucatán. Consideramos que su utilización contribuirá de forma positiva en el aprendizaje de temas matemáticos relacionados con la Geometría Euclidiana.
\end{abstract}

Palabras clave: Aprendizaje matemático; aplicación móvil; realidad aumentada.

\section{Introducción}

Los Resultados obtenidos por el Programa para la Evaluación Internacional de Alumnos (PISA) en el 2015, muestran que los alumnos mexicanos alcanzaron un promedio de 408 puntos en matemáticas estando por debajo del OCDE (Organización para la Cooperación y el Desarrollo Económico) de 490 [9]. Esto evidencia la necesidad de hacer un cambio en el proceso de enseñanza - aprendizaje que mejore el desempeño académico en los estudiantes mexicanos en el área de matemáticas. Para lograr lo anterior, se debe considerar algunos factores que afectan el aprendizaje del estudiante. En algunos casos se debe al temor de estudiar las asignaturas con contenido matemático por la complejidad de los conceptos, entrando en un estado de tensión y

\footnotetext{
Sosa-Jimenez, E., López-Martínez J., Chi-Pech,V.

Facultad de Matemáticas, UADY

Mérida, México.

Email: eduardo.sosa2819@gmail.com, jose.lopez@correo.uady.mx, victor.chi@correo.uady.mx

Sosa-Tzec, O.

Penny W. Stamps School of Art and Design, University of Michigan Ann Arbor, USA.

Email: omarsosa@umich.edu
}

frustración por parte del estudiante [3]. Las tecnologías de la información y de la comunicación (TIC) han jugado un papel muy importante en el sector educativo, estos han sido utilizados ampliamente en el aula para mejorar el proceso de enseñanza y aprendizaje en el estudiante [2]. Una de las herramientas tecnológicas más utilizadas recientemente es la Realidad Aumentada (RA), la cual ha demostrado su efectividad dentro del sector educativo como apoyo didáctico para la realización de actividades académicas eficaces apoyando el proceso cognitivo del estudiante [5]. Incorporar la RA en el proceso de enseñanzaaprendizaje no implica necesariamente sustituir los libros utilizados durante las clases. Su uso es para complementar y enriquecer la experiencia de aprendizaje con un ambiente atractivo y divertido que mejoraría la atención del estudiante [8].

\section{Propuesta de diseño}

En este proyecto, consideramos la fase de diseño como el momento crucial para discutir sobre la futura experiencia de usuario y sus diferentes elementos [4]. En el diseño de interacción y experiencia de usuario, una herramienta común para la ideación y evaluación de ideas es el bocetaje [6]. Este proyecto involucró dos rondas de bocetaje. En cada una, los bocetos funcionaron para materializar ideas, discutir sobre la factibilidad del proyecto, y externalizar preocupaciones sobre cómo el usuario interactuaría con la aplicación a desarrollar. El primer conjunto de bocetos se basó en la información recopilada de la literatura relacionada con aplicaciones que utilizan la RA. Los resultados de la discusión basada en estos bocetos informaron la siguiente ronda, en la cual los investigadores discutieron a fondo sobre las características finales para continuar con las siguientes dos fases: verificar la factibilidad del proyecto con base en las tecnologías de RA existentes en el mercado y el desarrollo de la aplicación. Una vez desarrollada la aplicación, nuestra metodología considera realizar pruebas de usabilidad [7] con estudiantes nivel medio-superior de la península de Yucatán.

El estudiante usaría la aplicación para repasar conceptos vistos en una clase de matemáticas. Este proyecto, en calidad de piloto, se enfoca en el concepto de parábola. La aplicación iría de la mano con un cuadernillo de ejercicios (Fig 1.a). Ambos servirán de complemento a las lecciones recibidas durante la clase. La aplicación está pensada para trabajar con tabletas y fuera del aula. De igual manera, tiene como principal objetivo reforzar el aprendizaje mediante la construcción gráfica de parábolas e interacción con ellas. El estudiante podrá hacer uso de la aplicación para comparar visualmente el resultado de graficar una 
parábola manualmente en el cuadernillo con el resultado correcto, el cual será mostrado en la aplicación a través de realidad aumentada (Fig. 1.b). Además, el usuario podrá modificar los parámetros de esta última parábola y otras generadas por el sistema. La pantalla inicial de la aplicación está dividida en dos grandes áreas (Fig. 2.a). En la parte superior, la aplicación mostrará animaciones interactivas con el fin de agregar un elemento divertido y atractivo como parte de la experiencia de usuario [1], similar a los doodles mostrados en la página de inicio de Google. En la parte inferior se presenta el concepto de parábola al estudiante. Del lado izquierdo, la aplicación muestra una parábola con todos sus componentes. Del lado derecho se muestra información textual y una serie de videos sobre cada uno de dichos componentes. Este será visualizado al realizar un tap al componente de la gráfica elegida por el estudiante.

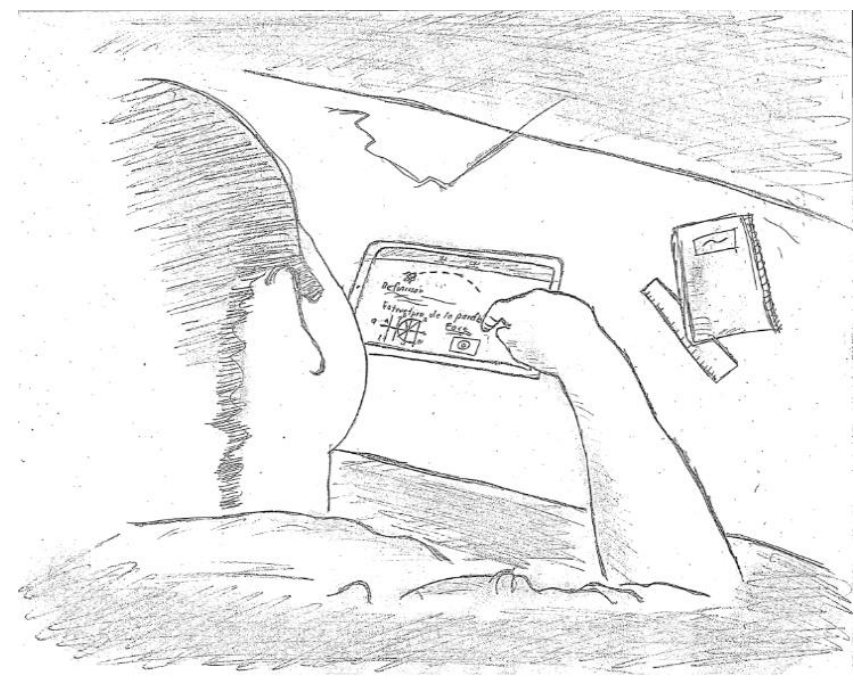

a)

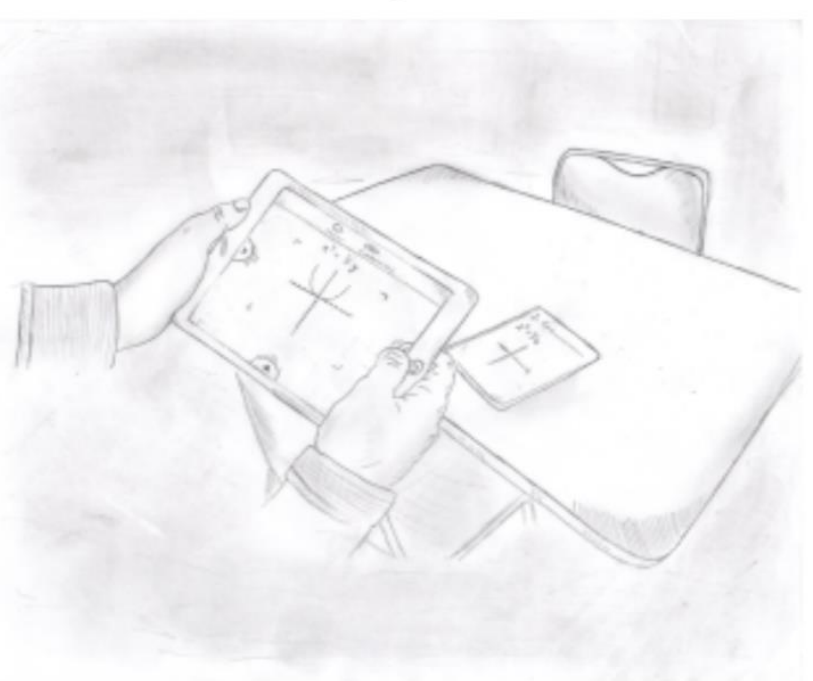

b)

Figure 1. (a) Representación del contexto de uso: aplicación y cuadernillo trabajarían en conjunto. (b) Uso de RA para comparar resultados de graficar manualmente

En la segunda pantalla (Fig.2.b), el estudiante podrá verificar los resultados de cada ejercicio presentado en el cuadernillo con el uso de la aplicación de RA. Dicha aplicación detecta la gráfica dibujada por el estudiante y simultáneamente se irá graficando el resultado correcto para después ser comparada. En esta misma pantalla, la aplicación le permite al usuario alterar los parámetros con el objetivo de generar nuevos resultados y ecuaciones que le darán al estudiante una mejor adquisición del conocimiento. Si el estudiante necesita una explicación más detallada de cómo resolver cada ejercicio, con un simple deslizamiento hacia la parte izquierda de la pantalla se desplegarán videos con el contenido necesario para el estudiante. La aplicación será capaz de generar múltiples ecuaciones de parábola para que el estudiante refuerce su aprendizaje a través de más comparaciones visuales. El estudiante puede seleccionar una nueva ecuación de parábola de una lista que será desplegada con un deslizamiento hacia la parte derecha de la pantalla, a partir de ahí dando un tap sobre la ecuación, ésta será graficada por la aplicación.

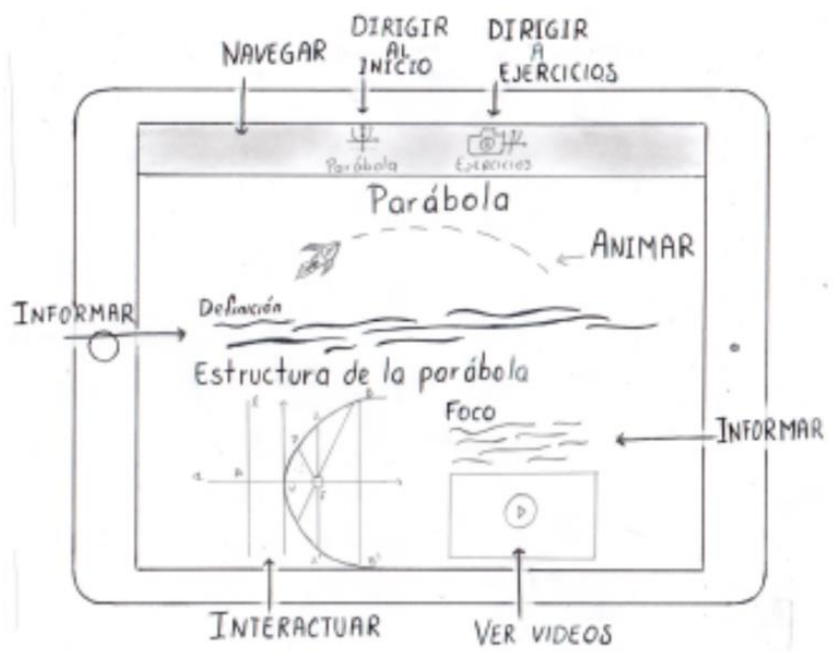

a)

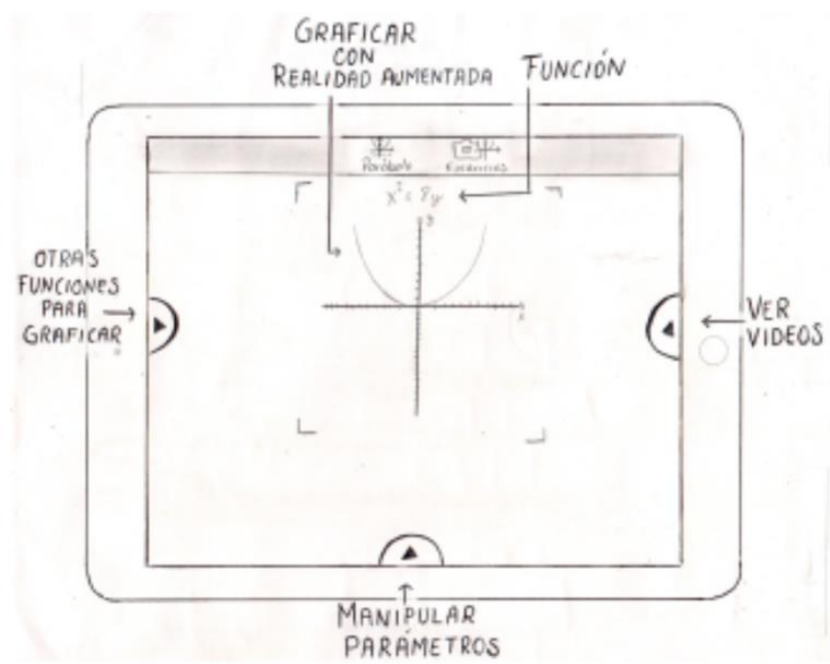

b)

Figure 2. (a) Pantalla inicial de la aplicación. (b) Pantalla de comparación de gráficas a través de RA.

\section{Conclusiones y trabajo a futuro}

Este trabajo propone una metodología para el diseño y desarrollo de una aplicación móvil con RA para coadyuvar en el aprendizaje 
de conceptos matemáticos. Los investigadores han decidido enfocarse en el concepto de parábola para llevar a cabo un proyecto piloto que ilustre dicha tecnología. Este trabajo describe las características y resultados de las dos primeras fases aplicadas a dicho proyecto. Este trabajo demuestra el valor del bocetaje como herramienta de diseño y discusión sobre la factibilidad del proyecto y elementos de la experiencia de usuario. Sin embargo, este trabajo también reconoce las limitaciones de esta herramienta, especialmente, la limitación de no poder informar acerca de cómo las características de la tableta afectarán el rendimiento de la aplicación y experiencia de usuario. Por este motivo, el proyecto enfocado en el concepto de parábola continuará con las siguientes fases, incluyendo el desarrollo de la aplicación y llevar a cabo las pruebas de usabilidad.

\section{Referencias}

[1] Mark A. Blythe, Kees Overbeeke, Andrew F. Monk, and C. Wright. Funology (1st ed.). Springer, Netherlands, Dordrecht, 2005.

[2] Cieza, E., and Lujan, D. Education Mobile Application of Augmented Reality Based on Markers to Improve the Learning of Vowel Usage and Numbers for Children of a Kindergarten in Trujillo. Procedia Computer Science, 130 (2018).

[3] Dodera, G. M., Bender, G., Burroni, E. A., Del, M., and Lázaro, P. Errores, actitud y desempeño matemático del ingresante universitario. Unión: revista iberoamericana de educación matemática (2014).

[4] Garrett, J.J. The elements of user experience: user-centered design for the Web and beyond (1st ed.). New Riders., Berkeley, CA, 2011.

[5] Jerabek, T., Rambousek, V., and Wildov, R. Specifics of Visual Perception of the Augmented Reality in the Context of Education. Procedia - Social and Behavioral Sciences 159 (2014), $598-604$.

[6] Löwgren, J., and Stolterman, E. Thoughtful Interaction Design: A Design Perspective on Information Technology. The MIT Press, 2004.

[7] Nielsen, J. Usability Engineering (1st ed.). Morgan Kaufmann Publishers Inc., San Francisco, CA, USA, 1994.

[8] Nincarean, D., Alia, M. B., Dayana, N., Halim, A., and Rahman., M. H. A. Mobile Augmented Reality: The Potential for Education. Procedia - Social and Behavioral Sciences 103 (2013), 657 - 664.

[9] Ramos, G., Schleicher, A., and González, C. Programa para la Evaluación Internacional del Alumno (PISA)-resultado. (2015). https://www.oecd.org/pisa/PISA-2015-MexicoESP.pdf 